\title{
Phantom scalar dark energy as modified gravity: understanding the origin of the Big Rip singularity
}

\author{
F. Briscese ${ }^{a}$, E. Elizalde ${ }^{(b) *}$, S. Nojiri ${ }^{(c) \dagger}$, and S. D. Odintsov ${ }^{(b, d) \ddagger}$ \\ (a) Dipartimento di Modelli e Metodi Matematici, \\ Università di Roma I, \\ Via A. Scarpa 16, I-00161, Roma, Italy \\ (b) Institut de Ciències de l'Espai (IEEC-CSIC) \\ Campus UAB, Facultat de Ciències, Torre C5-Parell-2a pl \\ E-08193 Bellaterra (Barcelona) Spain \\ (c) Department of Physics, \\ Nagoya University, Nagoya 464-8602, Japan \\ (d) Instituciò Catalana de Recerca i Estudis Avançats (ICREA), Barcelona, Spain
}

\begin{abstract}
It is shown that phantom scalar models can be mapped into a mathematically equivalent, modified $F(R)$ gravity, which turns out to be complex, in general. Only for even scalar potentials is the ensuing modified gravity real. It is also demonstrated that, even in this case, modified gravity becomes complex at the region where the original phantom dark energy theory develops a Big Rip singularity. A number of explicit examples are presented which show that these two theories are not completely equivalent, from the physical viewpoint. This basically owes to the fact that the physical metric in both theories differ in a time-dependent conformal factor. As a result, an FRW accelerating solution, or FRW instanton, in the scalar-tensor theory may look as a decelerating FRW solution, or a non-instantonic one, in the corresponding modified gravity theory.

PACS numbers: $11.25 .-\mathrm{w}, 95.36 .+\mathrm{x}, 98.80 .-\mathrm{k}$
\end{abstract}

\section{INTRODUCTION}

The explanation of the origin of dark energy and the precise description of the cosmological structure of the currently accelerating universe are fundamental challenges of modern cosmology. A good amount of observational data indicate quite clearly that the present universe may already be, or may soon enter, in a so-called phantom or superacceleration era, with an effective equation of state parameter $w$ slightly less than -1 (for a recent discussion on phantom-favoring observational data, see [1]). The simplest possibility to realize this phantom dark energy era is based on the introduction of a phantom scalar, i.e. a scalar field with negative kinetic energy (for a recent discussion of scalar phantom cosmology, see [2] and refs. therein). The fundamental property of such a phantom field in the accelerating FRW universe is the appearance of a finite-time future singularity (Big Rip) [3] of the scale factor. Moreover, a phantom scalar with negative kinetic energy leads to a number of instabilities and it is unwanted from a physical point of view.

In a situation like that, it is quite natural to search for other theories, without negative kinetic energy, which may also lead in a quite natural way to an effective phantom era. A rather straightforward possibility is modified gravity (for a recent review, see [4]), where indeed an effective phantom phase can be realized without a scalar phantom. It seems clear that the same phantom era can be alternatively described by modified gravity, by a phantom with some specific scalar potential, by a phantom-like ideal fluid, etc. Hence, it is important to investigate the relation between phantom scalar models and modified gravity, with the final aim to clarify what the different properties of both theories are.

It is known already that $F(R)$ modified gravity can be always presented under the mathematically-equivalent form of a (canonical) scalar-tensor theory, but this could not be proven for the scalar phantom one. In the present letter we will show that any scalar phantom theory can be always represented as a modified $F(R)$ gravity which is, generally speaking, complex but that for some potentials may be real. The class of phantom theories leading to real modified gravities will be investigated in some detail.

\footnotetext{
* elizalde@ieec.uab.es

† nojiri@phys.nagoya-u.ac.jp

‡ odintsov@ieec.uab.es, also at TSPU, Tomsk
} 
Before going on, it must be pointed out that, in spite of the mathematical equivalence of these two theories, they are not physically equivalent. This is due to the fact that the physical metric — which is to be fitted against the observational data - is different in both theories, owing to the appearance of a conformal factor in the process of transforming one theory into the other. As a result, an FRW instanton in one version is not necessarily an instanton in the (mathematically) equivalent theory, or an accelerating FRW cosmological solution in the scalar-tensor theory may result into a decelerating FRW universe, in the corresponding version of modified gravity. One must be very careful in analyzing all these possibilities. Furthermore, the origin of the Big Rip singularity will be hereby clarified, following the mapping of the phantom phase into real, modified gravity: the phantom Big Rip will precisely correspond to the region of modified gravity where it becomes complex.

\section{THE PHANTOM SCALAR AS (COMPLEX) MODIFIED GRAVITY}

Using a complex conformal transformation, we will show in this section how the phantom scalar can be represented as an equivalent modified gravity theory. The starting action for the scalar-tensor theory will be given by

$S=\int d^{4} x \sqrt{-g^{\prime}}\left\{\frac{R^{\prime}}{2 \kappa^{2}} \mp \frac{1}{2} \partial_{\mu} \varphi \partial^{\mu} \varphi-V(\varphi)\right\}$,

where $V(\varphi)$ is a potential of the scalar field $\varphi$ and $R^{\prime}$ is the scalar curvature corresponding to the metric tensor $g_{\mu \nu}$. In the above expression, the sign in front of the kinetic term is $+(-)$ for the case that the scalar field $\varphi$ is a phantom (canonical scalar). In [5] it has been shown that, in the non-phantom case, the scalar-tensor theory can be mapped - using a conformal transformation of the metric tensor - to modified gravity (for a general review of this procedure, see [4]). The relation between the two theories has been investigated with care. Mathematically, the two are equivalent but physically there are certain non-equivalency issues [5, 6, 6]. They are related with the fact that the physical metric that has to fit the observational data in the two theories is different (due to the conformal factor). For instance, some accelerating FRW universe solution of the scalar-tensor theory may well correspond to a decelerating FRW universe in the equivalent, modified gravity formulation (see some examples in [7]). Under these circumstances, if it turns out that the cosmological parameters are well fitted from the ones of the scalar-tensor theory, or either from those of modified gravity, it is this well behaved corresponding theory the one which will better describe our current accelerating universe. Note also that there was recent discussion [8] indicating that some versions of $F(R)$ gravity may have problems with Solar System tests. We will not discuss different points of view on this problem here.

A nontrivial problem occurs when a conformal transformation is used for the phantom case. Indeed, a real conformal transformation on the metric tensor of the type used in [5], $g_{\mu \nu}^{\prime}=\mathrm{e}^{ \pm \kappa \varphi \sqrt{\frac{2}{3}}} g_{\mu \nu}$, can cancel the kinetic term of the scalar field in the non-phantom case only. In order to solve this problem, we here suggest to use a complex conformal transformation which will lead to a (generally speaking, complex) modified $F(R)$ gravity.

From now on, we will restrict our discussion to the phantom scalar. To start, one can repeat step by step the calculations made in [5] by using a complex conformal transformation given by

$g_{\mu \nu}^{\prime}=\mathrm{e}^{ \pm i \kappa \varphi \sqrt{\frac{2}{3}}} g_{\mu \nu}$

Using it in (11), one arrives at

$S=\int d^{4} x \sqrt{-g}\left\{\frac{\mathrm{e}^{ \pm i \kappa \varphi \sqrt{\frac{2}{3}}} R}{2 \kappa^{2}}-\mathrm{e}^{ \pm i 2 \kappa \varphi \sqrt{\frac{2}{3}}} V(\varphi)\right\}$

where the kinetic term of the scalar field disappears. Now, the scalar $\varphi$ is just an auxiliary field and can be expressed in terms of the scalar curvature as $\varphi=\varphi(R)$, by using the equation of motion

$R=\mathrm{e}^{ \pm i \kappa \varphi \sqrt{\frac{2}{3}}}\left(4 \kappa^{2} V(\varphi) \mp i \sqrt{6} \kappa V^{\prime}(\varphi)\right)$

Hence, the scalar-tensor action appears under the form of $F(R)$ gravity:

$S=\int d^{4} x \sqrt{-g} F(R)$.

Here the function $F(R)$ is given by the expression

$F(R) \equiv \mathrm{e}^{ \pm i \kappa \varphi \sqrt{\frac{2}{3}}} \frac{R}{2 \kappa^{2}}-\mathrm{e}^{ \pm i 2 \kappa \varphi \sqrt{\frac{2}{3}}} V(\varphi)$. 
Note that, generally speaking, the curvature and the action itself can easily become complex, as they may contain a non-zero imaginary part after performing the complex conformal transformation. This fact indicates to known physical problems of phantom from another side.

Let us consider the simple example where the potential is given by

$V(\varphi)=V_{0} \mathrm{e}^{a k \varphi}$

Then, we find

$R=2 \kappa^{2} V_{0}\left(2 \mp i \sqrt{\frac{3}{2}} a\right) \mathrm{e}^{\kappa \varphi(R)\left(a \pm i \sqrt{\frac{2}{3}}\right)}$,

and

$F(R)=V_{0}\left(1 \mp i a \sqrt{\frac{3}{2}}\right)\left(\frac{R}{2 \kappa^{2} V_{0}\left(2 \mp i a \sqrt{\frac{3}{2}}\right)}\right)^{\frac{a \pm 2 i \sqrt{\frac{2}{3}}}{a \pm i \sqrt{\frac{2}{3}}}(1+2 n \pi i)}$.

As is clear from (4), if the scalar field $\varphi$ is real, the scalar curvature $R$ will not be always real. In order for $R$ to be real, the following condition should be fulfilled

$\mathrm{e}^{i \kappa \varphi \sqrt{\frac{2}{3}}}\left(4 \kappa^{2} V(\varphi)-i \sqrt{6} \kappa V^{\prime}(\varphi)\right)=\mathrm{e}^{-i \kappa \varphi \sqrt{\frac{2}{3}}}\left(4 \kappa^{2} V(\varphi)+i \sqrt{6} \kappa V^{\prime}(\varphi)\right)$

which is satisfied for a potential like

$V(\varphi)=\frac{V_{0}}{\cos \left(\kappa \varphi \sqrt{\frac{2}{3}}\right)}$.

Except for the case (11), the curvature $R$ in (4) is not real for real $\varphi$. There still could be, however, the possibility that, after formally solving (4) with respect to $\varphi$, if we substitute the expression into (3), the resulting action might be real. Let us assume that the potential $V(\varphi)$ contains only even powers of $\varphi$, and write this potential as $V(\varphi)=U\left(\varphi^{2}\right)$. This is a typical situation in quantum field theory (note that some properties of the phantom are indeed similar to those of a QFT, as was shown in [9], while quantum effects may render an effective phantom cosmology [10]). Since Eq.(14) can be rewritten as

$R=\mathrm{e}^{ \pm i \kappa \varphi \sqrt{\frac{2}{3}}}\left(4 \kappa^{2} U\left(\varphi^{2}\right) \mp i 2 \sqrt{6} \kappa U^{\prime}\left(\varphi^{2}\right) \varphi\right)$

this tells us that $\varphi$ is pure imaginary, if $R$ is real. Then, from the expression of (6), we find that the action (5) is indeed real. As $\varphi$ is purely imaginary, we can write it as $\varphi=i \phi$ and use the real field $\phi$. Then, even if we start with the plus sign in front of the kinetic term of the scalar field, in (1) — which corresponds to a phantom-it turns out that

$S=\int d^{4} x \sqrt{-g^{\prime}}\left\{\frac{R^{\prime}}{2 \kappa^{2}}-\frac{1}{2} \partial_{\mu} \phi \partial^{\mu} \phi-U\left(-\phi^{2}\right)\right\}$,

which corresponds to a non-phantom theory. Therefore, even if we started with a phantom theory, in order that the $F(R)$ gravity action could be real, the corresponding scalar-tensor theory reduces to a non-phantom theory, except for the case (11). We should note, however, that in the action (13), by analytic continuation, the potential becomes sometimes negative, as we will see later. This might be taken as a footprint of the original phantom nature of the theory. One further remark is in order. Some time ago complex general relativity attracted considerable interest, for different reason. In any case, if one starts with complex modified gravity where the whole imaginary part of the metric may be included into the conformal factor, then by an inverse transformation, such complex modified gravity can be mapped into a phantom scalar-tensor theory.

The next simple example is the model of a massive phantom and a cosmological constant:

$S=\int d^{4} x \sqrt{-g^{\prime}}\left\{\frac{R^{\prime}}{2 \kappa^{2}}+\frac{1}{2} \partial_{\mu} \varphi \partial^{\mu} \varphi-\frac{2 \alpha^{2}}{\kappa^{2}}-6 \alpha^{2} \varphi^{2}\right\}$.

Here $\alpha$ is a constant. A cosmological solution is given by

$a=a_{0} \mathrm{e}^{\alpha^{2} t^{2}}, \quad \varphi=\frac{2 \alpha t}{\kappa}$ 
Since $\dot{H}=2 \alpha^{2}>0$, the solution (15) actually expresses a super-accelerated (phantom) expanding (if $t>0$ ) universe. For the potential (14), Eq.(4) tells us that $\varphi$ is purely imaginary. In fact, when we define $\phi=-i \varphi$, Eq.(4) becomes real

$R=\mathrm{e}^{\mp \kappa \phi}\left(8 \alpha^{2}\left(1-3 \kappa^{2} \phi^{2}\right) \pm 12 \sqrt{6} \alpha^{2} \kappa \phi\right)$,

and can be solved as $\phi=\phi(R)$, and then we have the following $F(R)$ theory, from (6),

$F(R)=\frac{\mathrm{e}^{\mp i \kappa \varphi \sqrt{2 / 3}} R}{2 \kappa^{2}}-\frac{2 \alpha^{2} \mathrm{e}^{\mp 2 \sqrt{2 / 3} i \kappa \phi(R)}\left(1-3 \kappa^{2} \phi(R)^{2}\right)}{\kappa^{2}}$.

By inversely transforming the $F(R)$ gravity into the scalar-tensor theory, with the usual procedure, instead of (14), one obtains

$S=\int d^{4} x \sqrt{-g^{\prime}}\left\{\frac{R^{\prime}}{2 \kappa^{2}}-\frac{1}{2} \partial_{\mu} \phi \partial^{\mu} \phi-\frac{2 \alpha^{2}}{\kappa^{2}}+6 \alpha^{2} \phi^{2}\right\}$.

As the mass is negative, the scalar field is a tachyon. The action does not yield the same solution as (15), but if we Wick-rotate the time-coordinate $t$ as $t \rightarrow i \tau$, we obtain the Euclidean solution

$a=a_{0} \mathrm{e}^{-\alpha^{2} \tau^{2}}, \quad \phi=\frac{2 \alpha \tau}{\kappa}$.

We should point out that, in $F(R)$ gravity, owing to the scale transformation (2), the metric looks rather different

$d s_{F(R)}^{2}=\mathrm{e}^{ \pm i \kappa \varphi \sqrt{\frac{2}{3}}}\left(d \tau^{2}+a(\tau)^{2} \sum_{i=1}^{3}\left(d x^{i}\right)^{2}\right)=d \tilde{\tau}^{2}+\frac{2 a_{0}^{2} \alpha^{2} \tilde{\tau}^{2}}{3} \mathrm{e}^{-\frac{3}{2}\left(\ln \left(\mp \sqrt{\frac{2}{3}} \alpha \tilde{\tau}\right)\right)^{2}} \sum_{i=1}^{3}\left(d x^{i}\right)^{2}$.

Here

$\tilde{\tau} \equiv \mp \sqrt{\frac{3}{2}} \frac{\mathrm{e}^{\mp \sqrt{\frac{2}{3}} \alpha \tau}}{3}$.

The metric (20) has a conical singularity unless

$2 a_{0}^{2} \alpha^{2}=3$.

The third example is

$S=\int d^{4} x \sqrt{-g^{\prime}}\left\{\frac{R^{\prime}}{2 \kappa^{2}}+\frac{1}{2} \partial_{\mu} \varphi \partial^{\mu} \varphi-V_{0} \cos \left(\kappa \varphi \sqrt{\frac{2}{3}}\right)\right\}$.

By solving (4), we find

$\mathrm{e}^{ \pm 2 i \kappa \varphi \sqrt{2 / 3}}=-3+\frac{R}{V_{0} \kappa^{2}}$,

and therefore $\varphi$ is clearly imaginary. The corresponding $F(R)$ gravity (6) is given by

$F(R)=V_{0} \sqrt{-3+\frac{R}{V_{0} \kappa^{2}}}$.

Since $\varphi$ is imaginary, by putting $\varphi=-i \phi$, the action (23) acquires the following form:

$S=\int d^{4} x \sqrt{-g^{\prime}}\left\{\frac{R^{\prime}}{2 \kappa^{2}}-\frac{1}{2} \partial_{\mu} \phi \partial^{\mu} \phi-V_{0} \cosh \left(\kappa \phi \sqrt{\frac{2}{3}}\right)\right\}$,

which can be also obtained by inversely transforming the $F(R)$ gravity (25) into the scalar-tensor theory with the above procedure. In (26), when $|\phi|$ is large, the action behaves as

$S \sim \int d^{4} x \sqrt{-g^{\prime}}\left\{\frac{R^{\prime}}{2 \kappa^{2}}-\frac{1}{2} \partial_{\mu} \phi \partial^{\mu} \phi-\frac{V_{0}}{2} \mathrm{e}^{\kappa|\phi| \sqrt{\frac{2}{3}}}\right\}$. 
Hence, we have a solution like

$\phi \sim \kappa \sqrt{\frac{2}{3}} \ln \left|\frac{t}{t_{1}}\right|, \quad H=\frac{3}{t}, \quad t_{1} \equiv \frac{48}{\kappa^{2} V_{0}}$.

Since from Eq.(24) it follows that

$R=V_{0} \kappa^{2}\left(3+\mathrm{e}^{2 \kappa \phi \sqrt{\frac{2}{3}}}\right)$,

we find $R \rightarrow 3 V_{0} \kappa^{2}$ or $R \rightarrow+\infty$ when $|\phi| \rightarrow \infty$.

Thus, we have shown that a phantom scalar may be always mapped into complex modified gravity. In cases of an even scalar potential, the corresponding, equivalent modified gravity is real.

\section{THE BIG RIP SINGULARITY: PHANTOM VERSUS MODIFIED GRAVITY}

In the present section we will compare what happens in the phantom scalar-tensor theory and in modified gravity when a finite-time singularity (Big Rip) appears in either of these theories. Let us consider the following example

$S=\int d^{4} x \sqrt{-g^{\prime}}\left\{\frac{R^{\prime}}{2 \kappa^{2}}+\frac{1}{2} \partial_{\mu} \varphi \partial^{\mu} \varphi-V_{0} \cosh \left(2 \frac{\varphi}{\varphi_{0}}\right)\right\}$

Since $V_{0} \cosh \left(2 \frac{\varphi}{\varphi_{0}}\right) \sim \frac{V_{0}}{2} \mathrm{e}^{2|\varphi| / \varphi_{0}}$, when $\varphi$ is large, we have the following asymptotic solution

$\varphi \sim \varphi_{0} \ln \left|\frac{t_{0}-t}{t_{1}}\right|, \quad H \sim \frac{\kappa^{2} \varphi_{0}^{2}}{4\left(t_{0}-t\right)}, \quad t_{1}^{2} \equiv \frac{\varphi_{0}^{2}\left(1+\frac{3 \kappa^{2} \varphi_{0}}{4}\right)}{v_{0}}$,

which exhibits a Big Rip singularity [3] at $t=t_{0}$ (for the classification of future, finite-time singularities, see [11]). Hence, Eq.(44) yields

$R=\mathrm{e}^{ \pm i \kappa \varphi \sqrt{\frac{2}{3}}}\left(4 \kappa^{2} \cosh \frac{2 \varphi}{\varphi_{0}} \mp i \frac{2 \sqrt{6} \kappa}{\varphi_{0}} \sinh \frac{2 \varphi}{\varphi_{0}}\right)=\mathrm{e}^{\mp \kappa \phi \sqrt{\frac{2}{3}}}\left(4 \kappa^{2} \cos \frac{2 \phi}{\varphi_{0}} \mp \frac{2 \sqrt{6} \kappa}{\varphi_{0}} \sin \frac{2 \phi}{\varphi_{0}}\right)$.

Solving (32) with respect to $i \varphi$ or $\phi$ and using (5) and (6), we obtain an $F(R)$ gravity. Since the Big Rip singularity corresponds to $|\varphi| \rightarrow \infty$, the scalar curvature (32) in $F(R)$ gravity becomes complex and large as in (8). In particular, when $\phi \rightarrow+\infty$, one finds

$R \propto \mathrm{e}^{\left( \pm i \kappa \sqrt{\frac{2}{3}}+\frac{2}{\varphi_{0}}\right) \varphi}$

which gives a complex $F(R)$ theory with

$F(R) \propto R^{2-\frac{\frac{2}{\varphi_{0}}}{ \pm i \kappa \sqrt{\frac{2}{3}}+\frac{2}{\varphi_{0}}}}$.

Therefore, there is no solution in the corresponding $F(R)$ gravity which could be also obtained from a non-phantom theory

$S=\int d^{4} x \sqrt{-g^{\prime}}\left\{\frac{R^{\prime}}{2 \kappa^{2}}-\frac{1}{2} \partial_{\mu} \phi \partial^{\mu} \phi-V_{0} \cos \left(2 \frac{\phi}{\varphi_{0}}\right)\right\}$.

The point corresponding to the Big Rip singularity only appears when $\phi$ is analytically continued to be imaginary. This clearly demonstrates the physical non-equivalence between the phantom and the corresponding modified gravity theories: even when the (phantom) scalar-tensor theory can be mapped into a real $F(R)$ gravity, the FRW accelerating solution of the scalar-tensor theory might be mapped into the corresponding FRW solution in the $F(R)$ theory only partially. When the scalar-tensor FRW metric becomes singular (the Big Rip occurs) the equivalent $F(R)$ gravity becomes complex and the singularity does not show up. This is a quite general situation in the examples we have discussed. Nevertheless, one may also expect that in some specific cases the transformation of the scalar-tensor theory into modified $F(R)$ gravity may become singular precisely at the point where the Big Rip occurs. The generic conclusion is that when the phantom Big Rip occurs, there is no possibility to transform the Big Rip region of the scalar-tensor theory to a reliable (real) modified gravity sector. 
Conversely, the Big Rip singularity can occur even in $F(R)$ gravity, for instance, if [4]

$F(R)=f_{0} \mathrm{e}^{R / 6 H_{0}^{2}}$,

with constant $f_{0}$ and $H_{0}[4]$. We now consider what could occur in the corresponding scalar-tensor theory. Let us rewrite the general action of $F(R)$ gravity (5) as a scalar-tensor theory. By introducing the auxiliary fields, $A$ and $B$, one can rewrite the action (5) as follows

$S=\int d^{4} x \sqrt{-g}\left[\frac{1}{\kappa^{2}}\{B(R-A)+F(A)\}\right]$.

Then, one is able to eliminate $B$, to obtain

$S=\int d^{4} x \sqrt{-g}\left[\frac{1}{\kappa^{2}}\left\{F^{\prime}(A)(R-A)+F(A)\right\}\right]$,

and using the conformal transformation $g_{\mu \nu} \rightarrow \mathrm{e}^{\sigma} g_{\mu \nu}\left(\sigma=-\ln F^{\prime}(A)\right)$, the action (38) can be rewritten as the Einstein-frame action

$S_{E}=\int d^{4} x \sqrt{-g}\left[\frac{1}{\kappa^{2}}\left(R-\frac{3}{2} g^{\rho \sigma} \partial_{\rho} \sigma \partial_{\sigma} \sigma-V(\sigma)\right)\right]$.

Here,

$V(\sigma)=\mathrm{e}^{\sigma} G\left(\mathrm{e}^{-\sigma}\right)-\mathrm{e}^{2 \sigma} f\left(G\left(\mathrm{e}^{-\sigma}\right)\right)=\frac{A}{F^{\prime}(A)}-\frac{F(A)}{F^{\prime}(A)^{2}}$.

The action (38) is called the Jordan-frame action (a recent comparison of the equivalence between the Einstein and the Jordan frames can be found in [12]). If we identify $\varphi=\sqrt{3} \sigma / \kappa$, we obtain the action of the scalar-tensor theory (11). The action thus obtained is not the phantom one, since the scalar field in (40) has a canonical kinetic term. Near the Big Rip singularity, the scalar curvature in $F(R)$ gravity becomes large and then, for the model (36), we find $\sigma \propto-R / 6 H_{0}^{2}$. As a consequence, $\sigma$ becomes negative and large, and the potential $V(\sigma) \rightarrow 0$.

When $F(R)$ behaves as $F(R) \sim R^{-n}$, the scalar factor behaves as

$a \sim\left(t_{0}-t\right)^{\frac{(n+1)(2 n+1)}{n+2}}$.

Thus, when $n<-2$ or $-1<n<-1 / 2$, a singularity of the Big Rip type can appear at $t=t_{0}$. In this case, we find

$\sigma \sim(n+1) \ln R \sim-2(n+1) \ln \left(t_{0}-t\right)$,

since

$R \sim \frac{6(n+1)(2 n+1)(4 n+5) n}{(n+2)^{2}\left(t_{0}-t\right)^{2}}$.

Then, in the corresponding scalar-tensor theory, the time coordinate $\tilde{t}$ could be given by $d \tilde{t}= \pm \mathrm{e}^{\sigma} d t \sim \pm\left(t_{0}-t\right)^{n+1} d t$, that is $\tilde{t}= \pm\left(t_{0}-t\right)^{n+2}$. Therefore, when $n<-2, t \rightarrow t_{0}$ corresponds to $t \rightarrow \pm \infty$. As a consequence, the singularity changes its structure: it does not appear in finite time for the scalar-tensor theory. On the other hand, when $n>-2$, $t \rightarrow t_{0}$ corresponds to $t \rightarrow 0$. We also find that the metric in the scalar-tensor theory behaves as

$d s_{S T}^{2}=\mathrm{e}^{\sigma}\left(-d t^{2}+a(t)^{2} \sum_{i=1,2,3}\left(d x^{i}\right)^{2}\right) \sim-d \tilde{t}^{2}+\tilde{a}(t)^{2} \sum_{i=1,2,3}\left(d x^{i}\right)^{2}, \quad \tilde{a}(t)^{2} \sim a_{0}^{2} \tilde{t}^{\frac{(n+1)(2 n+5)}{(n+2)^{2}}}$,

with a constant $a_{0}$. Since in the case $-1<n<-1 / 2, a(t)^{2} \rightarrow 0$ when $\tilde{t} \rightarrow 0$, the Big Rip singularity could be replaced with a Big Crunch, where the universe shrinks indefinitely.

Hence, the Big Rip singularity which may occur in some versions of $F(R)$ gravity may change its structure in the equivalent, scalar-tensor theory. Either it goes to the infinite past or future $(n<-2)$, or the Big Rip singularity is replaced with a Big Crunch singularity $(-1<n<-1 / 2)$ in the corresponding scalar-tensor theory. Thus, generically, there is in fact a mathematical equivalence between the phantom scalar-tensor theory and the corresponding modified gravity, the Big Rip singularity region being then the part of the solution where physical equivalence is lost. This may be due to the actual non-existence of one of the corresponding theories precisely in this region, or either to a total change of the structure and properties of the singularity. 


\section{THE FRW INSTANTON WITH A SPATIALLY NON-FLAT METRIC BOTH IN THE PHANTOM THEORY AND IN MODIFIED GRAVITY}

In Sect. 2 we have studied the analytic continuation of the phantom scalar field, while in [13], the reconstruction scenario for the scalar-tensor theory was considered. In this formulation the scalar field is identified with the time coordinate. As we analytically continue the scalar field to pure imaginary values (Sect. 2), the time coordinate could be also analytically continued. Then one could obtain a kind of an instanton solution. In [13], only the case that the spatial part is flat was considered, since the spatial part of the observed universe is approximately flat. In order to obtain a finite action for the instanton solution, however, we may consider the case when the spatial part is spherical. But even if the spatial part is flat or a hyperboloid, dividing the manifold by using a discrete group, we can also obtain a finite action. In either way, we are able to extend the formulation of [13] to the (phantom) case when the spatial part is not flat

$d s^{2}=-d t^{2}+a(t)^{2} d \Omega^{2}$.

Here $d \Omega^{2}$ is the metric of either the three-dimensional flat space, the hyperboloid, or the sphere with unit radius. The action of the scalar-tensor theory is chosen to be

$S=\int d^{4} x \sqrt{-g}\left\{\frac{R}{2 \kappa^{2}}-\frac{1}{2} \omega(\varphi) \partial_{\mu} \phi \partial^{\mu} \phi-V(\phi)\right\}$,

By assuming that the scalar field $\varphi$ only depends on time, the FRW equations give

$0=-\frac{3}{\kappa^{2}}+\frac{1}{2} \omega^{\phi} \dot{\phi}^{2}+V(\phi)-\frac{3 k}{2 \kappa^{2} a^{2}}, \quad 0=\frac{1}{\kappa^{2}}\left(\dot{H}+3 H^{2}\right)+\frac{1}{2} \omega^{\phi} \dot{\phi}^{2}-V(\phi)+\frac{k}{2 \kappa^{2} a^{2}}$.

If $d \Omega^{2}$ in (45) is the metric of the sphere, we have $k=2$, if it corresponds to a hyperboloid, $k=-2$, and in the flat case $k=0$. Since there is freedom in redefining the scalar field $\phi$, we may choose $\phi=t$. Then, we obtain

$\omega(\phi)=-\frac{2}{\kappa^{2}} \dot{H}+\frac{k}{\kappa^{2} a^{2}}, \quad V(\phi)=\frac{1}{\kappa^{2}}\left(\dot{H}+3 H^{2}\right)+\frac{k}{\kappa^{2} a^{2}}$.

As a consequence, if we consider the model where $\omega(\phi)$ and $V(\phi)$ are given by

$\omega(\phi)=-\frac{2}{\kappa^{2}} g^{\prime \prime}(\phi)+\frac{k \mathrm{e}^{-2 g(\phi)}}{\kappa^{2} a_{0}^{2}}, \quad V(\phi)=\frac{1}{\kappa^{2}}\left(g^{\prime \prime}(\phi)+3 g^{\prime}(\phi)^{2}\right)+\frac{k \mathrm{e}^{-2 g(\phi)}}{\kappa^{2} a_{0}^{2}}$,

there is the following solution

$\phi=t, \quad H=g^{\prime}(t) \quad\left(a=a_{0} \mathrm{e}^{g(t)}\right)$.

First we consider a special (and trivial) example with $k=2$ (sphere):

$g(\phi)=\ln \cosh \frac{\phi}{a_{0}}$.

Then by using (50), we find that the metric is given by

$d s^{2}=-d t^{2}+a_{0} \cosh \frac{t}{a_{0}} d \Omega^{2}$,

which is nothing but the metric of deSitter space. In fact, (48) yields

$\omega(\phi)=0, \quad V(\phi)=\frac{3}{a_{0}^{2} \kappa^{2}}$.

Therefore, the scalar field $\phi$ does not appear in this action (46), there only appears the cosmological term, where the cosmological constant is given by $V$ in (53). As well-known, by Wick-rotating the time coordinate $t$ by $t \rightarrow i a_{0} \tau$, the metric (52) is transformed into the metric of the sphere with radius $a_{0}$ :

$d s^{2}=a_{0}^{2}\left(d \tau^{2}+\cos ^{2} \tau d \Omega^{2}\right)$.

The solution (54) can be regarded as an instanton. 
Let us consider the second non-trivial example, again when $k=2$ :

$g(t)=\ln \left\{\frac{1}{2}\left(1+\frac{t^{2}}{a_{0}^{2}}\right)\right\}$,

which gives

$\omega(\phi)=\frac{4}{\kappa^{2} a_{0}^{2}}\left(1+\frac{\phi^{2}}{a_{0}^{2}}\right)^{-2}, \quad V(\phi)=\frac{10}{a_{0} \kappa^{2}}\left(1+\frac{\phi^{2}}{a_{0}^{2}}\right)^{-1}$.

Eq. (55) tells us that

$a(t)=\frac{a_{0}}{2}\left(1+\frac{t^{2}}{a_{0}^{2}}\right)^{2}$.

We may analyticaly continue the scalar field $\phi$ as $\phi=i \rho$, which gives

$\omega(\rho)=\frac{4}{\kappa^{2} a_{0}^{2}}\left(1-\frac{\rho^{2}}{a_{0}^{2}}\right)^{-2}, \quad V(\rho)=\frac{10}{a_{0} \kappa^{2}}\left(1-\frac{\rho^{2}}{a_{0}^{2}}\right)^{-1}$.

In the Euclidean signature, $t=i \tau$, the scalar-tensor theory leads to the following metric, instead of (57),

$d s^{2}=d \tau^{2}+\frac{a_{0}}{2}\left(1-\frac{\tau^{2}}{a_{0}^{2}}\right)^{2} d \Omega^{2}$

This metric seems to be singular when $\tau \rightarrow \pm a_{0}$. Since the metric behaves as

$d s^{2} \sim d \tau^{2}+\left(\tau-a_{0}\right)^{2} d \Omega^{2}$,

when $\tau \rightarrow-a_{0}$ and

$d s^{2} \sim d \tau^{2}+\left(a_{0}-\tau\right)^{2} d \Omega^{2}$

when $\tau \rightarrow a_{0}$, there is no conical singularity when $\tau \rightarrow \pm a_{0}$. Therefore, this metric (59) corresponds to the instanton.

By changing the scalar field $\phi$ to $\tilde{\phi}$ as

$\phi=a_{0} \tan \left(\frac{\kappa}{2} \tilde{\phi}\right)$

Eqs.(58) yield the following action

$S=\int d^{4} x \sqrt{-g}\left\{\frac{R}{2 \kappa^{2}}-\frac{1}{2} \partial_{\mu} \tilde{\phi} \partial^{\mu} \tilde{\phi}-\frac{10}{a_{0}^{2} \kappa^{2}} \cos ^{2}\left(\frac{\kappa}{2} \tilde{\phi}\right)\right\}$,

and by analytic continuation of $\tilde{\phi}$ as $\tilde{\phi}=i \varphi$, the action is transformed into

$S=\int d^{4} x \sqrt{-g}\left\{\frac{R}{2 \kappa^{2}}+\frac{1}{2} \partial_{\mu} \varphi \partial^{\mu} \varphi-\frac{10}{a_{0}^{2} \kappa^{2}} \cosh ^{2}\left(\frac{\kappa}{2} \varphi\right)\right\}$

which corresponds to a phantom field. Since, from (62)

$\rho=a_{0} \tanh \left(\frac{\kappa}{2} \varphi\right)$

the action (64) gives the metric in (59). If we start with the action (56), Eqs.(4) and (6) lead to a corresponding $F(R)$ gravity theory. Even if we start with the action (63), we obtain the same $F(R)$ gravity action. More explicitly, Eq. (44) gives

$R=\frac{20 \mathrm{e}^{ \pm \kappa \tilde{\phi} \sqrt{\frac{2}{3}}}}{a_{0}^{2}}\left(2 \cos (\kappa \tilde{\phi})+2 \mp \sqrt{\frac{3}{2}} \sin (\kappa \tilde{\phi})\right)$.

In $F(R)$ gravity, the Lorentz-signature metric has the following form:

$d s_{F(R)}^{2}=\mathrm{e}^{\mp i \sqrt{\frac{2}{3}} \ln \left(\frac{a_{0}+i t}{a_{0}-i t}\right)}\left(-d t^{2}+\frac{a_{0}}{2}\left(1+\frac{t^{2}}{a_{0}^{2}}\right)^{2} d \Omega^{2}\right)$,

which seems difficult to continue analytically in the Euclidean signature.

All the above shows that the properties of the Euclidean-signature solution in $F(R)$ gravity are quite different from those in the corresponding (phantom) scalar-tensor theory. Therefore, this indicates again a certain physical non-equivalence of $F(R)$ gravity as compared to the corresponding (phantom) scalar-tensor theory. Note also that the conclusions of the previous section about the Big Rip singularity do not change for the case of the spatially non-flat FRW universe. 


\section{DISCUSSION}

In summary, we have here demonstrated that any phantom scalar theory can be mapped into a mathematically equivalent one, which is a complex modified gravity. The fact that the mathematically-equivalent theory is complex can be taken, generically speaking, as an indication of some problems (already well known in fact) concerning the physical properties of the phantom field.

For even scalar potentials, the ensuing modified gravity turns out to be real. Nevertheless, even in this case it becomes complex in the region where the scale factor develops the well-known Big Rip singularity. Thus, the correspondence we have unveiled helps a lot to clarify the origin of the Big Rip singularity.

From a different perspective, we have also seen that, when some version of $F(R)$ gravity develops an effectively phantom universe, with a possible future Big Rip, the corresponding scalar-tensor theory is not a phantom one. Moreover, the structure of the Big Rip in the modified gravity changes to some other type of singularity in the scalartensor theory: it usually becomes an infinite-time one or has a totally different nature. Even a transmutation from a Big Rip to a Big Crunch singularity is possible.

As a final remark, let us recall the known fact that any (canonical or phantom) scalar-tensor theory can be presented, in an equivalent form, as a fluid obeying some equation of state. This equivalence can then easily be extended to modified gravity [5]. As a result, using our connection here, an ideal fluid phantom dark energy model might be also presented as modified $F(R)$ gravity, and the comparison of the properties of both theories is to be performed in a way similar to what we have done in this work.

\section{Acknowledgements}

This investigation was completed during a stay of FB in Barcelona. It has been supported in part by MEC (Spain), projects BFM2003-00620 and PR2006-0145, by JSPS (Japan) XXI century COE program of Nagoya University project 15COEEG01, by Monbu-Kagaku-sho grant no.18549001 (Japan), by LRSS project N4489.2006.02, by RFBR grant 06-01-00609 (Russia), and by AGAUR (Generalitat de Catalunya), contract 2005SGR-00790.

[1] S. Nesseris and L. Perivolaropoulos, arXiv:astro-ph/0610092 H. Jassal, J. Bagla and T. Padmanabhan, arXiv:astro-ph/0601389 P. Wu and H. Yu, arXiv:astro-ph/0611507; Z. Huang, Q. Sun, W. Fang and H. Lu, hep-th/0612176.

[2] V. Faraoni, Phys. Rev. D 68 (2003) 063508; Class. Quant. Grav. 22 (2005) 3235; E. Elizalde, S. Nojiri and S. D. Odintsov, Phys. Rev. D 70 (2004) 043539 arXiv:hep-th/0405034; S. Nojiri and S. D. Odintsov, Phys. Lett. B 595 (2004) 1 arXiv:hep-th/0405078; Phys. Lett. B 571 (2003) 1 arXiv:hep-th/0306212; B. Gumjudpai, T. Naskar, M. Sami and S. Tsujikawa, JCAP 0506 (2005) 007; S. Nesseris and L. Perivolaropoulos, Phys. Rev. D 70 (2004) 123529; Y. Wei, Mod. Phys. Lett. A 20 (2005) 1147; P. Gonzalez-Diaz, TSPU Vestnik 44N7 (2004) 36 arXiv:hep-th/0408225]; W. Fang, H. Lu and Z. Huang, arXiv:hep-th/0604160 Z. Huang et al., arXiv:hep-th/0610019, L. P. Chimento and R. Lazkoz, Int. J. Mod. Phys. D 14 (2005) 587; I. Arefeva and I. Volovich, arXiv:hep-th/0612098; S. Tsujikawa and M. Sami, Phys. Lett. B 603 (2004) 113; Z. Guo, Y. Piao, X. Zhang and Y. Zhang, arXiv:astro-ph/0608165 M. Alimohammadi and H. Mohseni, Phys. Rev. D 74 (2006) 043506; M. Setare, Phys. Lett B 641 (2006) 130; S. Vernov, astro-ph/0612487; S. Tsujikawa, Phys.Rev. D 73 (2006) 103504; M. Sami and A. Toporensky, Mod.Phys.Lett. A 19 (2004) 1509; P. Wu and H. Yu, Int. J. Mod. Phys. D 14 (2005) 1873.

[3] B. McInnes, JHEP 0208 (2002) 029.

[4] S. D. Odintsov, S. Nojiri, arXiv:hep-th/0601213; arXiv:hep-th 0611071.

[5] S. Capozziello, S. Nojiri and S. D. Odintsov, Phys. Lett. B 634 (2006) 93 arXiv:hep-th/0512118; S. Nojiri and S. D. Odintsov, Phys. Rev. D 68 (2003) 123512 arXiv:hep-th/0307288.

[6] T. Sotiriou, Class. Quant. Grav. 23 (2006) 5117.

[7] S. Capozziello, S. Nojiri, S.D. Odintsov and A. Troisi, Phys. Lett. B 639 (2006) 135 arXiv:astro-ph/0604431.

[8] T. Chiba, T. Smith, and A. Erickcek, arXiv:astro-ph/0611867; G. Olmo, arXiv:gr-qc/0612047 X. Jin, D. Liu and X. Li, arXiv:astro-ph/0610854.

[9] S. Nojiri and S. D. Odintsov, Phys. Lett. B 562 (2003) 147 arXiv:hep-th/0303117.

[10] V. K. Onemli and R. Woodard, Phys. Rev. D 70 (2004) 107301; E. Kahya and V. Onemli, gr-qc/0612026

[11] S. Nojiri, S. D. Odintsov and S. Tsujikawa, Phys. Rev.D 71 (2005) 063004 arXiv:hep-th/0501025.

[12] V. Faraoni and S. Nadeau, arXiv:gr-qc/0612075.

[13] S. Nojiri, S. D. Odintsov, Gen. Rel. Grav. 38 (2006) 1285 arXiv:hep-th/0506212; S. Capozziello, S. Nojiri, S. D. Odintsov, Phys. Lett. B 632 (2006) 597 arXiv:hep-th/0507182; S. Nojiri, S. D. Odintsov, H. Stefancic, Phys. Rev. D 74 (2006) 086009 arXiv:hep-th/0608168. 